\title{
Rapid Earthquake Characterization Using MEMS Accelerometers and Volunteer Hosts Following the $M 7.2$ Darfield, New Zealand, Earthquake
}

\author{
by Jesse F. Lawrence, Elizabeth S. Cochran, Angela Chung, Anna Kaiser, \\ Carl M. Christensen, Richard Allen, Jack W. Baker, Bill Fry, Thomas \\ Heaton, Deborah Kilb, Monica D. Kohler, and Michela Taufer
}

\begin{abstract}
We test the feasibility of rapidly detecting and characterizing earthquakes with the Quake-Catcher Network (QCN) that connects low-cost microelectromechanical systems accelerometers to a network of volunteer-owned, Internet-connected computers. Following the 3 September 2010 M 7.2 Darfield, New Zealand, earthquake we installed over 180 QCN sensors in the Christchurch region to record the aftershock sequence. The sensors are monitored continuously by the host computer and send trigger reports to the central server. The central server correlates incoming triggers to detect when an earthquake has occurred. The location and magnitude are then rapidly estimated from a minimal set of received ground-motion parameters. Full seismic time series are typically not retrieved for tens of minutes or even hours after an event. We benchmark the QCN real-time detection performance against the GNS Science GeoNet earthquake catalog. Under normal network operations, QCN detects and characterizes earthquakes within $9.1 \mathrm{~s}$ of the earthquake rupture and determines the magnitude within 1 magnitude unit of that reported in the GNS catalog for $90 \%$ of the detections.
\end{abstract}

\section{Introduction}

Over the past decade, several cyber-social-seismic networks have been developed, including the Personal Seismic Network (Cranswick et al., 1993), NetQuakes (Luetgert et al., 2009), the Quake-Catcher Network (QCN; Cochran, Lawrence, Christensen, and Chung, 2009; Cochran, Lawrence, Christensen, and Jakka, 2009), and the Community Seismic Network (Clayton et al., 2011). New sensor technology and computational techniques provide an avenue for creating very large cyber-social-seismic networks by reducing instrument costs, minimizing needed infrastructure, and harnessing public interest. Small low-cost (\$30-\$3000) microelectromechanical systems (MEMS) triaxial sensors provide ground-acceleration measurements of moderate to large earthquakes (Cochran, Lawrence, Christensen, and Chung, 2009; Cochran, Lawrence, Christensen, and Jakka, 2009; Chung et al., 2011; Cochran et al., 2011). Data from these low-cost sensors are transmitted to a central server either through an Internetconnected computer or via any available wireless connection (Luetgert et al., 2009; Cochran, Lawrence, Christensen, and Chung, 2009; Cochran, Lawrence, Christensen, and Jakka, 2009; Clayton et al., 2011). These networks minimize the costs associated with monitoring the sensors by utilizing the host's computing resources, A/C power, Internet, and shelter (Luetgert et al., 2009; Cochran, Lawrence, Christensen, and Chung, 2009; Cochran, Lawrence, Christensen, and Jakka, 2009; Clayton et al., 2011).

The QCN represents one type of cyber-social-seismic network. In the QCN architecture, MEMS sensors are connected directly to Universal Serial Bus (USB) ports on a host's computer; the computer monitors the sensor and sends time series and ground-motion parameters to a central server. This is a low-cost paradigm compared to traditional sensor networks and even other cyber-social-seismic networks such as the NetQuakes program (Luetgert et al., 2009), which places self-contained independent systems in homes and offices. The USB sensors used by QCN range in cost from $\$ 30$ to $\$ 150$. Since QCN's inception in 2007, the network has grown to over 2000 volunteers worldwide. The stations are distributed globally (Cochran, Lawrence, Christensen, and Chung, 2009; Cochran, Lawrence, Christensen, and Jakka, 2009) as well as in dense arrays in particular regions of interest, including the rupture zones of major earthquakes to monitor aftershock sequences (Chung et al., 2011; Cochran et al., 2011).

Berkeley Open Infrastructure for Network Computing (BOINC), a cyber-infrastructure software package (Anderson and Kubiatowicz, 2002; Anderson, 2004; Christensen et al., 2005), is used to initiate and manage communication between a host computer and the central server (Cochran, Lawrence, 
Christensen, and Chung, 2009; Cochran, Lawrence, Christensen, and Jakka, 2009). BOINC allows hosts to donate any desired portion of their CPU and Internet bandwidth to a variety of scientific projects (Anderson, 2004). Hosts also provide a USB port and 15-30 min of their time to install the sensor and report the sensor's location through a Google Map online interface. Sensors are typically shipped to volunteers through the mail or may be transported via luggage on commercial airlines, particularly for rapid aftershock deployments.

The MEMS sensors used by the QCN network have steadily improved since the network began in 2007. The noise floor of the MEMS accelerometers has decreased from $4 \times 10^{-2} \mathrm{~m} / \mathrm{s}^{2}$ in 2008 to $2 \times 10^{-3} \mathrm{~m} / \mathrm{s}^{2}$ in 2010 , and $<6 \times 10^{-4} \mathrm{~m} / \mathrm{s}^{2}$ for the sensors currently used (Cochran, Lawrence, Christensen, and Chung, 2009). MEMS accelerometer amplitude and phase responses are relatively flat from 0 to $10 \mathrm{~Hz}$ (Holland, 2003; Farine et al., 2004; Evans et al., 2013). The digitizers have improved from 8-bit in 2008-2010 (Cochran, Lawrence, Christensen, and Chung, 2009), to 14-bit in 2010-2011 (Cochran et al., 2011, and data used in this study), to 16-bit since 2011. As a result of MEMS accelerometer and digitizer improvements, the lowest-magnitude earthquake recorded with reasonable fidelity through $\mathrm{QCN}$ has dropped from $M 4.5$ in 2008 (Cochran, Lawrence, Christensen, and Chung, 2009) to $M 2.6$ in 2010.

In 2010 QCN initiated a Rapid Aftershock Deployment Program (RAMP) following the $M 8.8$ Maule, Chile, earthquake (Chung et al., 2011). A set of earthquake detection, location, and characterization algorithms were developed in retrospective tests using the continuous aftershock dataset (Chung et al., 2011). The results of that study suggested that moderate to large earthquakes could be detected, located, and their magnitudes estimated using a limited set of ground-motion parameters transmitted to the central server from the QCN stations. For events reported by both QCN and the U.S. Geological Survey National Earthquake Information Center (USGS NEIC), the retrospective tests resulted in similar estimated locations, magnitudes, and shaking intensities (Chung et al., 2011).

Here, we describe the data collected during a RAMP initiated following the M 7.2 2010 Darfield, New Zealand, earthquake. Specifically, we report the results of real-time rapid earthquake detections using only parametric data provided by QCN stations. The emphasis here is on testing the performance of the rapid earthquake detection algorithms and the feasibility of using social-cyber-seismic network data for earthquake characterization.

\section{Data}

On 3 September 2010, an $M 7.2$ earthquake occurred in Darfield, New Zealand, located just northwest of the urban center of Christchurch (Gledhill et al., 2010). QCN and GNS Science collaborated to initiate a RAMP to augment the existing GNS strong-motion network with USB accelerometers (Cochran et al., 2011). Volunteers were recruited to host (a)

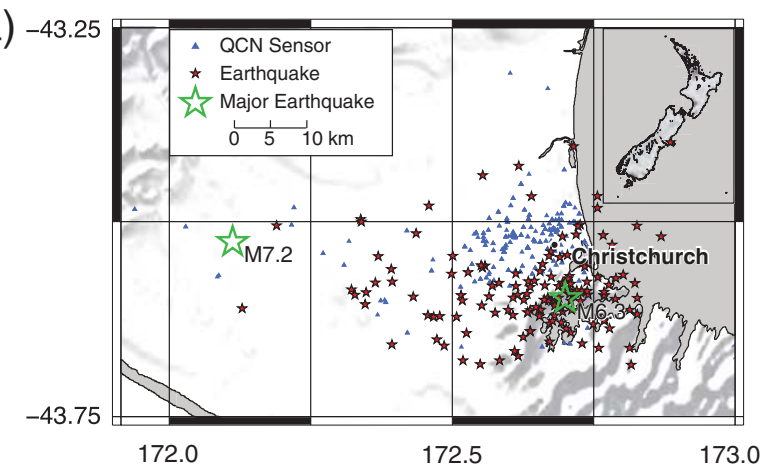

(b)
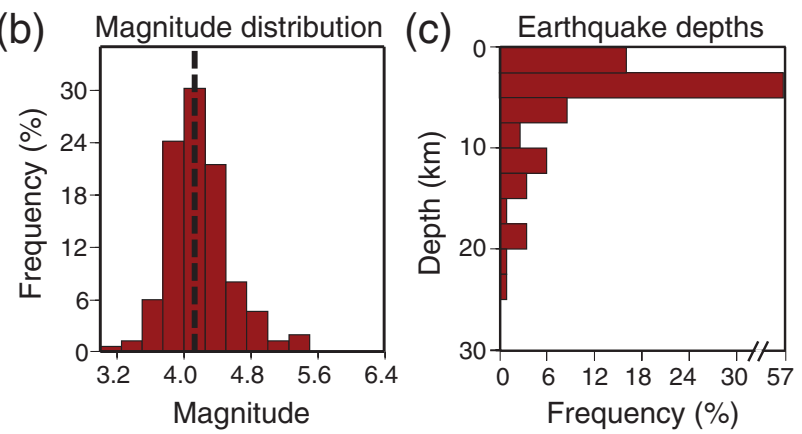

Figure 1. Seismic sensors and earthquakes detected by the lowcost sensor network between 25 September 2010 and 1 April 2011. (a) Map of sensors (blue triangles), detected earthquakes (red stars), and major earthquakes (green stars); inset map shows the study area (red star). (b) Magnitude distribution and (c) depth distribution of detected earthquakes.

QCN sensors through local media outlets. Within 11 days of the mainshock, over 180 QCN sensors were deployed in homes in and around Christchurch (Fig. 1). Note that the placement of sensors was limited by availability of hosts. Therefore, the stations tend to be located in population centers rather than distributed evenly around the aftershock sequence. The majority of sensors were aligned, leveled, and mounted to the ground floors or basements of residences. Data were recorded at 50 samples per second. See Cochran, Lawrence, Christensen, and Chung, 2009, Cochran, Lawrence, Christensen, and Jakka, 2009, and Chung et al. (2011) for additional details of the network architecture. Cochran et al. (2011) showed that closely spaced QCN and GeoNet stations provide similar peak acceleration, velocity, and displacement time series. And, although the QCN sensors are lower resolution and recorded fewer events, they found that the GeoNet and QCN stations provide comparable peak ground accelerations (PGAs) and peak ground velocities (PGVs) as well as root mean square scatter in the observations.

QCN stations can record in either continuous or triggered mode. The New Zealand RAMP stations were set up to record in continuous mode, with time series uploaded to the central server in 10-minute-long packets. Even in continuous mode, the host computer monitors the three component data for significant ground motion using a variant of a short-term average over long-term average (STA/LTA; e.g., Earle and Shearer, 

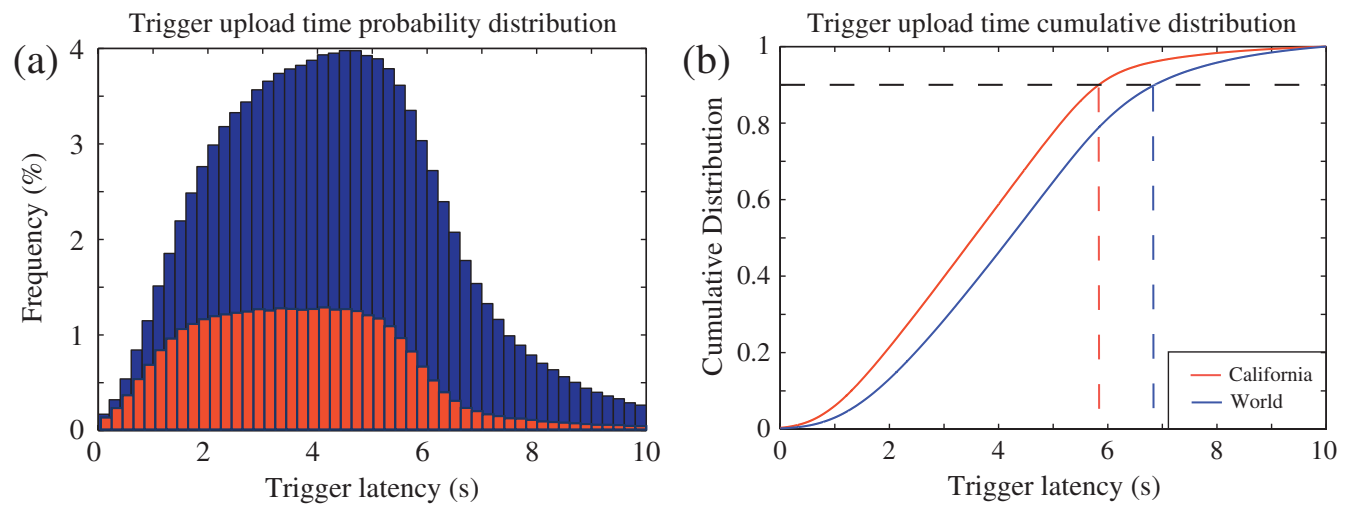

Figure 2. Latencies (s) for triggers detected on volunteers' computers to be transferred to the QCN central server located in California. (a) Histogram and (b) cumulative distribution of trigger latencies. Blue indicate latencies from all QCN stations distributed globally and red are latencies for stations in California. Dashed black line indicates the cumulative distribution of 0.9.

1994) to generate triggers (Chung et al., 2011). QCN replaces the LTA with a long-term standard deviation $\left(\sigma_{\mathrm{LT}}\right)$. The ratio STA $/ \sigma_{\mathrm{LT}}$ is the statistical definition of signal-tonoise ratio (SNR) and is the inverse to the coefficient of variation (a measure of dispersion; McDonough and Whalen, 1995). This formulation of SNR is only applicable for nonnegative numbers, so we apply the statistic to the vector magnitude of demeaned acceleration. QCN uses a $0.06 \mathrm{~s}$ short-term window and a 60 s long-term window. We require that the SNR exceed the $99 \%$ confidence interval $(\sim 3)$ for a trigger to be issued. To reduce multiple triggers associated with one strong new signal, we require that all triggers have STA values and SNR ratios $10 \%$ greater than any STA and SNR values determined in the previous second.

Following a trigger, the maximum acceleration at the time of the trigger is determined. The PGAs defined here differs from traditional measurements. The traditional PGA is not ideal for rapid event detection because it requires waiting until the entire wavetrain from an event has passed by a station before issuing a measurement. Therefore, we introduce a variant of PGA at a given time lag $(\tau)$ : PGA $\tau$. This time lag is relative to a trigger, for example, the detection time of a strong new motion. The peak acceleration at the time of the trigger, $\tau=0$, is given by PGA0. The peak acceleration up to $4 \mathrm{~s}$ following the trigger is denoted by PGA4.

Further, we use the maximum vector magnitude of the acceleration, $|\mathbf{a}|$, rather than assessing the horizontal or vertical magnitudes separately. We demean each component prior to taking the magnitude of the vector. The peak vector magnitude of acceleration $\mathrm{PGA}_{|\mathbf{a}|}$ is typically dominated by the peak horizontal acceleration, $\mathrm{PGA}_{\mathrm{H}}$. Note that $\mathrm{PGA}_{|\mathbf{a}|}$ removes the partitioning of energy between vertical and horizontal, which may vary due to site conditions, source mechanism, and earth structure. In some cases within this paper, we refer to $\mathrm{PGA}_{|\mathbf{a}|}$ and in other cases we refer to $\mathrm{PGA}_{|\mathbf{a}|} \tau$. The peak vector magnitude accelerations at the time of the trigger and up to $4 \mathrm{~s}$ following the trigger are given by $\mathrm{PGA}_{|\mathbf{a}|} 0$ and $\mathrm{PGA}_{|\mathbf{a}|} 4$. We note that near the source, PGA and $\mathrm{PGA}_{|\mathbf{a}|} 4$ are typically very similar.
Trigger information and ground-motion parameters derived from the time series are transferred to the servers in real time. However, there is a finite time delay between the time of an initial detection on the volunteer computer and when that trigger is registered in the $\mathrm{QCN}$ database. This latency accrues as the trigger information and associated data are saved to the volunteer computer, the trigger information is uploaded to the central server, and the server parses and registers the trigger information. Figure 2 illustrates the probability and cumulative distribution functions for the trigger latencies of sensors in California and worldwide. The mean latencies are $\sim 3.4$ and $4.2 \mathrm{~s}$ for California and worldwide, respectively, and over $90 \%$ of all triggers are registered within 6 and $7 \mathrm{~s}$ for California and worldwide, respectively. There are several factors that may contribute to lower latencies within California, including proximity to the QCN server located in California, faster average Internet connections, and/or computers with greater processing capabilities.

For less than $0.09 \%$ of triggers, latencies are greater than $10 \mathrm{~s}$. In some instances, Internet dropouts can cause triggers to be delayed by up to several days. In such cases, the trigger data are clearly not useful for rapid earthquake detection and characterization. Following a major earthquake it is possible for power and Internet to fail, causing the network to lose sensitivity. Power and Internet outages following the $M_{\mathrm{w}} 6.321$ February 2011 Christchurch earthquake reduced the number of QCN sensors reporting aftershocks for $\sim 48 \mathrm{hrs}$. This is a critical limitation of QCN; however, traditional networks may also lose contact with stations during major events (e.g., Kilb et al., 2007).

\section{Rapid Earthquake Detection}

On 25 September 2010, QCN implemented preliminary real-time earthquake detection and characterization using only trigger information from the dense QCN network in Christchurch. The rapid earthquake detection algorithm monitors the triggers registered by the real-time database, including the trigger time, peak vector amplitude of acceleration at 


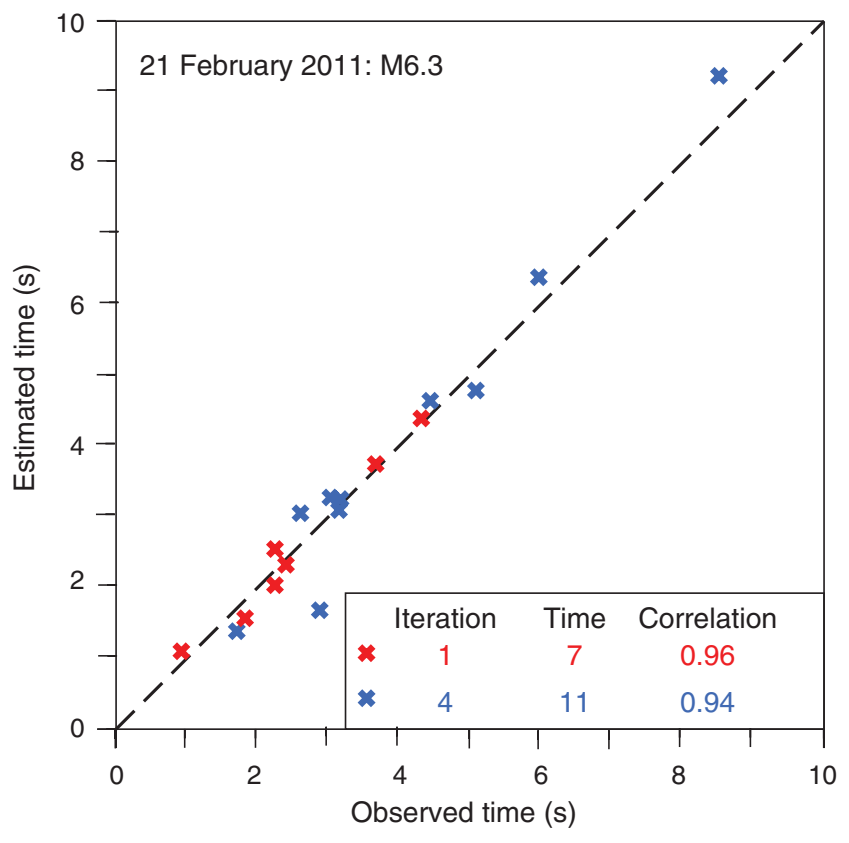

Figure 3. Correlation of modeled travel times to observed travel times for the $M 6.3$ earthquake on 21 February 2011, with values shown for detection iteration 1 (red) and 4 (blue).

the time of the trigger (referred to here as $\mathrm{PGA}_{|\mathbf{a}|} 0$ ), and station information. The triggers transfer rapidly from the volunteer computer to the server (2-5 s; Fig. 2) through BOINC's trickle protocol. The initial transfer of compact data descriptions, rather than full waveforms, greatly reduces communication latencies between the host computer and central server.

Triggers are temporally and spatially correlated at the QCN server to evaluate if they are associated with a regional earthquake or due to isolated, single-station noise. We evaluate triggers received at $0.2 \mathrm{~s}$ intervals, comparing each trigger with all other triggers that occurred within the previous $100 \mathrm{~s}$. If triggers occur within $100 \mathrm{~s}$ and the stations are located within $200 \mathrm{~km}$ they are considered roughly correlated (Chung et al., 2011). We apply an SNR detection algorithm (as above) to the number of roughly correlated triggers with a minimum of $99 \%$ confidence and a minimum requirement of 5 correlated signals. Here, the short-term window is $100 \mathrm{~s}$ and the long-term window is 5 days.

Once the number of roughly correlated triggers exceeds the SNR (typically 6-7) or the minimum requirement (5), we attempt to locate the earthquake hypocenter using a 3D grid search (Chung et al., 2011). Further details of the earthquake location algorithm are available in Chung et al. (2011). Figure 3 shows the correlation between the estimated travel times and the observed travel times for the 21 February 2011 $M 6.3$ earthquake. The QCN hypocenters are within $\sim 7 \mathrm{~km}$ of the GNS reported locations, on average, which have reported errors on the order of $1 \mathrm{~km}$ in latitude and longitude. Differences in the estimated hypocenter locations may result from the higher noise level of the QCN sensors as well as different station distributions, seismic-velocity models, and

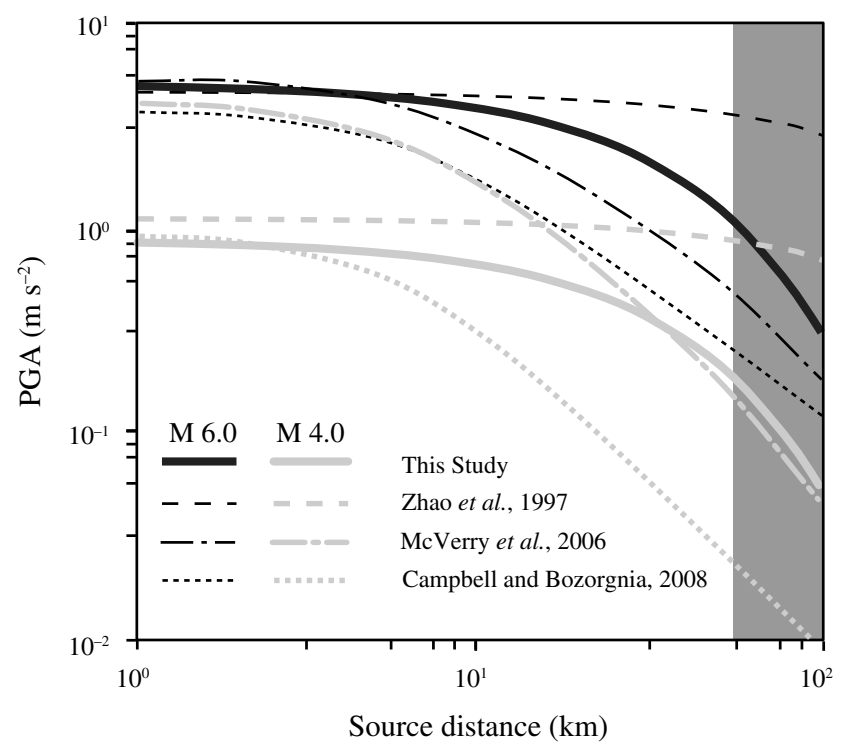

Figure 4. Comparison between the PGA relationship obtained here and the GMPEs of Zhao et al. (1997), McVerry et al. (2006), and Campbell and Bozorgnia (2008). Note the relationships deviate at greater distances; however, there are fewer QCN PGA measurements available to constrain the relationship at these distances (gray region).

location algorithms. The excellent correlation (e.g., Fig. 3, in which $R^{2}>0.94$ ) between observed and theoretical travel times for the best-fit source locations suggests the events are well located, given the a priori seismic-velocity model. Online accessible figures are automatically generated for each iteration based on the best-fit location determined by a 3D grid search. This correlation between observed and estimated travel times are used in the rapid earthquake location scheme. Only best-fit locations with correlations better than $R^{2}>0.5$ are considered probable earthquakes.

Next, we estimate the earthquake magnitude by fitting the PGA as a function of distance (e.g., Gutenberg and Richter, 1956; Donovan, 1973). The scatter in PGA is often quite high due to a regionally heterogeneous geologic material and the site conditions at each sensor (e.g., Cochran et al., 2011). However, PGA measured at multiple stations can provide a stable magnitude estimate. For the QCN New Zealand data, we derived the empirical relationship between magnitude, distance, and $\mathrm{PGA}_{|\mathbf{a}|}$ :

$$
\mathrm{M}=0.03 \Delta+1.09 \ln \left(\mathrm{PGA}_{|\mathbf{a}|}\right)+4.28,
$$

in which $\Delta$ is distance in $\mathrm{km}$ and $\mathrm{PGA}_{|\mathbf{a}|}$ is the vector sum of the three-component PGA in $\mathrm{m} / \mathrm{s}^{2}$. This relationship is similar to those previously reported for New Zealand crustal earthquakes using ground-motion prediction equations (GMPEs) of Zhao et al. (1997), McVerry et al. (2006), and Campbell and Bozorgina (2008), suggesting previously determined PGA-distance-magnitude relationships can be used (Fig. 4). Figure 4 shows the more typical PGA computed from the full waveform, rather than PGA $\tau$. The relationship 
(a)

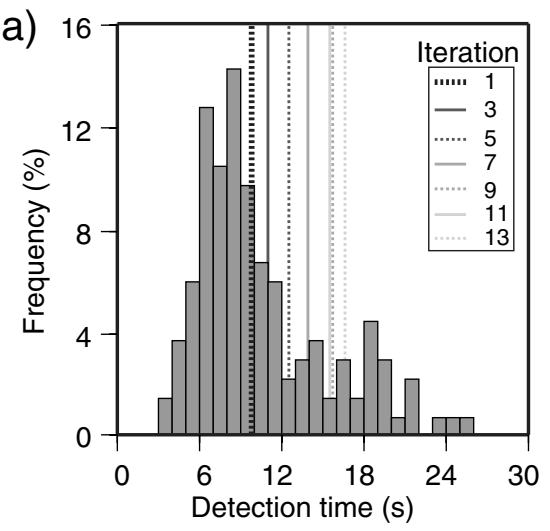

(d)

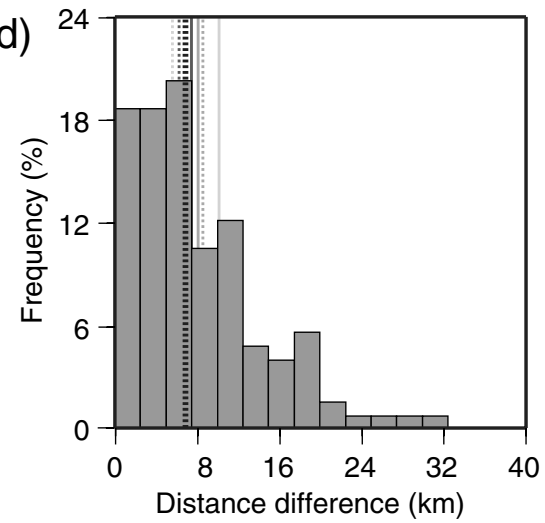

(b)

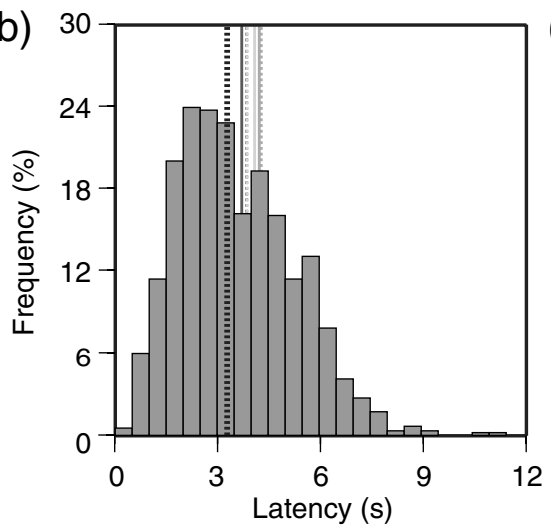

(e)

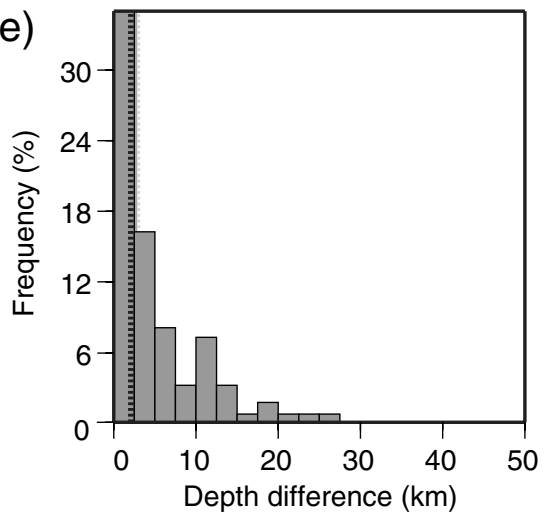

(c)

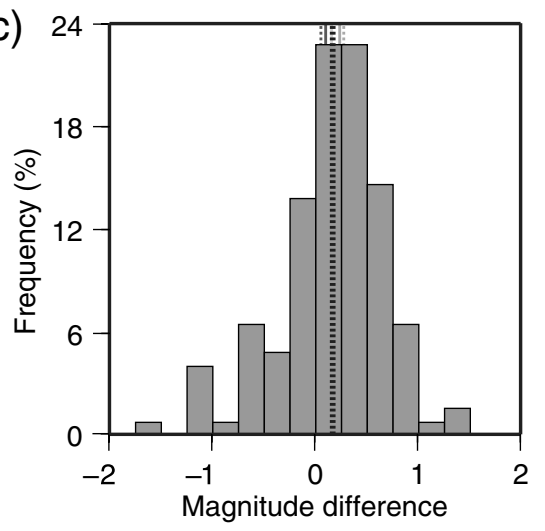

(f)

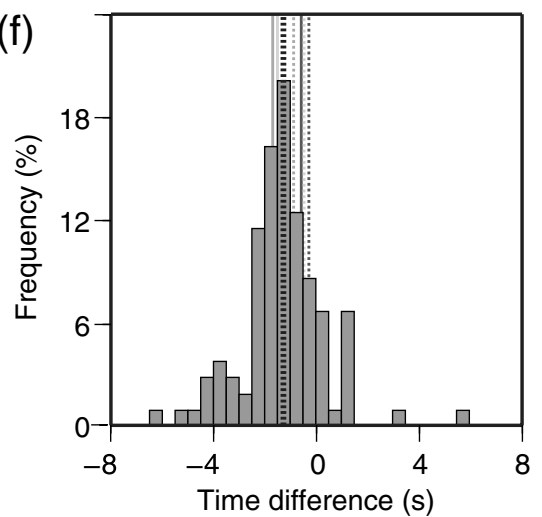

Figure 5. Rapid earthquake detection statistics for 116 earthquakes between 25 September 2010 and 1 April 2011. (a) Time between estimated earthquake origin to initial earthquake detection. (b) Latency from host signal detection to trigger registry on the QCN server. Histograms comparing QCN rapid earthquake characterization and GNS catalog of: (c) magnitude; (d) epicentral location; (e) depth; and (f) origin time (s). Histograms are shown for the first iteration; lines indicate median values for each iteration. The magnitudes are within 0.5 and 1.0 magnitude units for $63 \%$ and $90 \%$ of all earthquakes, respectively.

used here is within the range of previously reported GMPEs. Note that here we use vector PGA rather than the geometric mean of the horizontals more typically applied to GMPEs; however, the vertical ground motions only contribute minimally to the vector PGA. There are some differences in the GMPEs, which may be due to limited data quantities and scatter in measured accelerations due to source and site effects. At close distance ranges all of the models, including the model derived from QCN data, converge to similar PGAs. The majority of the ground-acceleration measurements available in the dataset used here are at source-to-station distances of 5-30 km.

QCN host computers transmit updated $\mathrm{PGA}_{|\mathbf{a}|}$ measurements at $\tau=1,2$, and $4 \mathrm{~s}$ after the initial trigger $\left(\mathrm{PGA}_{|\mathbf{a}|} 1-4\right)$, which are used to iteratively improve magnitude estimates. New data between each iteration may include new triggers $\left(\mathrm{PGA}_{|\mathbf{a}|} 0\right)$ reported by additional sensors or from new wave arrivals at the same sensor and updated $\mathrm{PGA}_{|\mathbf{a}|} \mid-4$ from stations that had previously triggered. The number of iterations for an earthquake depends on the earthquake location and magnitude relative to the distribution of seismic stations.

Between 25 September 2011 and 1 April 2011 the QCN sensors detected 116 earthquakes ranging in size from $M 3.6$ to $M 6.3$ (Figs. 1 and 5). The minimum and median detection times were 3.1 and $9.8 \mathrm{~s}$ after the estimated earthquake origin time, respectively (Fig. 5a). This time includes: (1) source-tosensor propagation (variable), (2) trigger detection $(<1 \mathrm{~s})$, (3) transfer of trigger information to the QCN server (2-5 s), (4) trigger association and event declaration (requires at least five associated triggers, variable), (5) event detection $(<0.1 \mathrm{~s})$, earthquake location $(<0.02 \mathrm{~s})$, magnitude estimation $(<0.01 \mathrm{~s})$, (6) AlertMap generation $(\sim 0.4 \mathrm{~s})$, and (7) posting to the web ( $\sim 0.4 \mathrm{~s}$ concurrent with AlertMap generation). Approximately half of the delay between the earthquake origin and detection is accounted for in wave propagation from the hypocenter to seven or more sensors ( $4.3 \mathrm{~s}$ on average). The median magnitude estimate increases by 0.5 magnitude units during the first four iterations, likely due to the fact that smaller earthquakes often result in only one or two iterations, whereas larger events typically result in a greater number of iterations because more sensors trigger with equal or greater $\mathrm{PGA}_{|\mathbf{a}|} 0-4$. However, some the observed increase in magnitude with number of iterations may be due to updated $\mathrm{PGA}_{|\mathbf{a}|} 0-4$ estimates which can increase as more of the wavefield is observed at a station, including the $S$ wave and subsequent coda.

To determine the accuracy of the real-time detection and characterization we compare the QCN earthquake catalog 
with the published GNS Science catalog. The accuracy/precision of the real-time estimates varied over time because of attrition of QCN volunteers, improvements to the monitoring process, and power and communications failures following large quakes. Although we conducted retrospective testing of the earthquake detection algorithms following the $M 8.8$ Maule, Chile, earthquake (Chung et al., 2011), the algorithms had not been tested in real time. We implemented a preliminary version of the real-time detection algorithms on 25 September 2011 and, over the following months, added features, improved algorithms, and expanded the real-time output features.

Because of the low-resolution nature of the QCN sensors, many ( 51) earthquakes with magnitude greater than $M 3.5$ located within $15 \mathrm{~km}$ of the array were not automatically detected. Continuous waveform records for most of the stations were uploaded to the central server, so data from the events not automatically detected were not lost. During the first 32 days, 11 false detections occurred, usually with a low number (5-6) of triggering stations. After several algorithm improvements implemented in October 2010, no false detections and characterizations were reported through April 2011. The most important algorithm change to eliminate false detections was to ignore triggers from a subset of the stations that repeatedly triggered due to chronic sensor or computer malfunction and/or extremely frequent local noise sources.

The network size reduced gradually throughout the deployment period (11 September 2010-1 April 2011) as hosts were initially recruited for an $\sim 6$ week period to record the early aftershock period following the 3 September 2011 $M$ 7.2 Darfield earthquake. Immediately prior to the $21 \mathrm{Feb}-$ ruary $2011 M 6.3$ Christchurch earthquake, only $40 \pm 10$ sensors were operating in the region. GNS Science redeployed some sensors following the February earthquake, bringing the network to $50 \pm 10$ sensors. As expected, the network capabilities diminish as the number of sensors decreased. Nevertheless, dozens of earthquakes were detected and recorded prior to the $M 6.3$ earthquake even with fewer sensors reporting.

Immediately following the 21 February $2011 M 6.3$ Christchurch earthquake the capabilities of the real-time detection were further reduced due to the following power outages and a rapid succession of aftershocks. Vigorous aftershock sequences can challenge even very robust networks (e.g., Kilb et al., 2007). QCN failed to detect 54 aftershocks with magnitude greater than $M 3.5$ in the 48 hrs following the $M 6.3$ Christchurch earthquake. With ongoing and planned algorithm changes to discriminate between event updates and new events, we expect fewer complications for future vigorous aftershock sequences; however, widespread power failures would still reduce or eliminate the ability to detect events.

We compare the origin time, hypocenter, and magnitude for the QCN detected events to the GeoNet catalog (Fig. 5). On average, we find that the median error in earthquake location is $\sim 7 \mathrm{~km}$, the QCN earthquake origin times are biased
$1.5 \mathrm{~s}$ early, and the magnitude estimates are $\sim 0.5$ magnitude units too high. These differences can be attributed primarily to different station distributions, velocity models, and attenuation relationships. Because of the limited distribution of available hosts, QCN sensors are clustered in a small region $\left(10 \times 10 \mathrm{~km}^{2}\right)$ with the majority of the earthquakes occurring outside of the network (Fig. 1). This tends to increase the location error, biasing earthquake locations farther from the network, and leading to overestimation of magnitude and earlier origin times (Fig. 5). However, given these limitations, the QCN rapid earthquake detections compare favorably to the GNS Science catalog.

QCN automatically generates an AlertMap of observed, interpolated, and predicted peak shaking intensities (e.g., Allen and Kanamori, 2003) for each detected earthquake. Here, intensity is estimated as instrumental intensity (Wald, Quitoriano, Heaton, and Kanamori, 1999; Wald, Quitoriano, Heaton, Kanamori, et al., 1999), using $\mathrm{PGA}_{|\mathbf{a}|} 0-4$ instead of PGA. For most of the events evaluated here, $\mathrm{PGA}_{|\mathbf{a}|} 0-4$ is similar to PGA because the earthquakes are small and recorded at short source-receiver distances; thus, the maximum accelerations occur within the first $4 \mathrm{~s}$ of the record. For large magnitude events and/or greater source-receiver distances, $\mathrm{PGA}_{|\mathbf{a}|} 0-4$ may underestimate the true instrumental intensity. AlertMaps are generated when the earthquake is first detected and improved with each iteration as additional data are reported. Because the median event detection time is approximately $10 \mathrm{~s}$, the AlertMap often provides a prediction of expected peak shaking intensities for locations away from the epicenter. Figure 6 shows an AlertMap of estimated peak ground-shaking intensities and the estimated time until shaking arrives at each location. The individual $\mathrm{PGA}_{|\mathbf{a}|} 0-4 \mathrm{ob}-$ servations suggest fine-scale variability in ground-shaking intensities. The smooth background contours provide an interpolated estimate of the ground-shaking intensities between observations and, for locations farther from the hypocenter, the predicted shaking.

\section{Discussion}

The results of the real-time earthquake detection and characterization tests using only ground-motion parameters derived from the temporary array of QCN stations are promising. The rate at which stations were installed (over 180 stations in 11 days) is ideal for recording and detecting aftershocks shortly after a mainshock; however, the station distribution is somewhat limited to urban areas based on available volunteers. The resources required to transport a large number of QCN sensors into the aftershock area were minimal compared to traditional RAMP experiments due to the lightweight and compact size of the QCN sensors.

The QCN network and real-time algorithms were able to determine the magnitude and location of events with median detection times of $9.1 \mathrm{~s}$ after the origin time. The primary delay is propagation of the $P$ waves from the source to 5-7 stations as well as latencies to transfer the ground-motion 
(a)

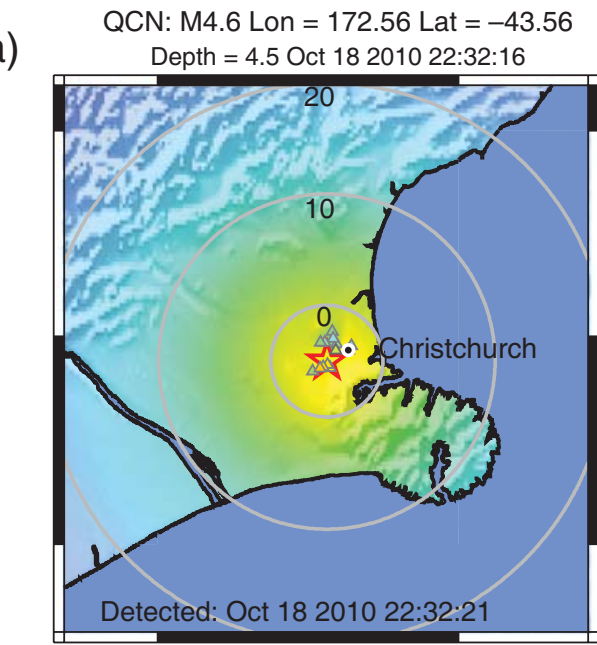

$172^{\circ}$

QCN: M4.6 Lon $=172.56$ Lat $=-43.56$

Depth $=4.5$ Oct 182010 22:32:16

$172^{\circ}$
\begin{tabular}{|c|c|c|c|c|c|c|c|c|c|}
\hline $\begin{array}{c}\text { Perceived } \\
\text { Shaking }\end{array}$ & Not Felt & Weak & Light & Moderate & Strong & Very Strong & Severe & Violent & Extreme \\
\hline $\begin{array}{c}\text { Potential } \\
\text { Damage }\end{array}$ & none & none & none & Very Light & Light & Moderage & Moderate/Heavy & Heavy & Very Heavy \\
\hline Peak Acc $(\% \mathrm{~g})$ & $<0.17$ & $0.17-1.4$ & $1.4-4.0$ & $4.0-9$ & $9-17$ & $17-32$ & $32-61$ & $61-114$ & $>114$ \\
\hline Peak Vel (cm/s) & $<0.12$ & $0.12-1.1$ & $1.1-3.4$ & $3.4-8$ & $8-16$ & $16-31$ & $31-59$ & $59-115$ & $>115$ \\
\hline $\begin{array}{c}\text { Instrumental } \\
\text { Intensity }\end{array}$ & $\mathrm{I}$ & II-III & III & V & VI & VII & VIII & IX & X+ \\
\hline
\end{tabular}

Figure 6. AlertMaps generated (a) $5 \mathrm{~s}$ and (b) $24 \mathrm{~s}$ after the earthquake origin time by QCN's rapid earthquake detection system for an $M 4.8$ Christchurch earthquake. The gray circles illustrate isochrons for $S$-wave arrival time relative to the detection time (positive times indicate expected future arrivals, negative times indicate waves have arrived). Initial AlertMaps are very similar to later iterations. The intensity scale is from (Wald, Quitoriano, Heaton, and Kanamori, 1999; Wald, Quitoriano, Heaton, Kanamori, et al., 1999).

parameters from the station to the central server over the Internet. Errors in the location $(\sim 7 \mathrm{~km})$ and magnitude $( \pm 1$ magnitude unit) are similar to errors from previously reported earthquake early warning algorithms (4-13 km and 0.5-0.75 magnitude units; e.g., Allen, 2006; Allen et al., 2009).

It is unclear how QCN's real-time algorithms will perform during large or great earthquakes $(>M$ 6.5) measured at close source-receiver distances $(<40 \mathrm{~km})$. PGA and other waveform measurements can saturate, resulting in an underestimate of magnitude (e.g. Kanamori, 2005). So, although use of PGA $\mathrm{Pa}_{|\mathbf{a}|} 0-4$ was largely successful in this experiment, other measurements, such as the maximum period of displacement used in earthquake early warning (EEW) algorithms, may be more reliable (e.g., Allen and Kanamori, 2003; Cua and Heaton, 2007; Allen et al., 2009). Nevertheless, retrospective application of these algorithms to the Maule, Chile, aftershock sequence provided accurate magnitude estimates for events up to $M 6.9$ (Chung et al., 2011).

The rapid updates to the location, magnitude, and AlertMaps for each detected event suggest that, as triggers from more distant stations are included, earthquake characterization improves regardless of PGA saturation. Dense networks such as the RAMP described here provide a unique opportunity to evaluate earthquakes in real time. Even as we test a variety of EEW parameters (e.g., Allen and Kanamori, 2003; Cua and Heaton, 2007; Allen et al., 2009), real-time knowledge of $\mathrm{PGA}_{|\mathbf{a}|} 0-4$ will likely provide useful information regarding the spatial distribution of ground-shaking intensities, which can deviate from the idealized distance-dependent models. Rapid estimates of ground-shaking intensities and the distribution of strong ground motions can be used to infer the true size of an event.

Because initial locations, magnitudes, and AlertMaps are generated within seconds of the earthquake origin, this information could be potentially used to provide an EEW alert to individuals or organizations of incoming strong ground motion (Hoshiba et al., 2008). The initial test of this notification system was conducted using a small pool of inhouse participants and no notifications were provided to the general public. Beginning 5 March 2011, QCN implemented a tool to automatically disseminate event information to a select group of users. The notification (sent via e-mail) included the earthquake metadata (origin time, location, time of detection, and magnitude) and an AlertMap. The notifications can be filtered based on input participant-defined preferences for epicentral distance and magnitude cutoff. These notifications were typically received by the in-house participants within $1 \mathrm{~s}$ of event detection. For this cursory test, we used Simple Mail Transfer Protocol (SMTP) (Hughes, 1998) and Internet Message Access Protocol (IMAP) (Heinlein and Hartleben, 2008). SMTP and IMAP are not ideal for immediate mass data dissemination, but the rudimentary test illustrated that earthquake information detected by the low-cost QCN network could be distributed rapidly.

With an average detection time of $9.1 \mathrm{~s}$, and an additional $\sim 1 \mathrm{~s}$ for issuing a notification, participants in New Zealand 
could have received alerts if such notifications had been issued to individuals external to QCN's personnel. For $\sim 83 \%$ of the aftershocks one or more participants around Christchurch could have received an alert before the strong surface waves hit (assuming an $\sim 3 \mathrm{~km} / \mathrm{s}$ surface-wave velocity). This statistic is skewed because three participants hosted sensors $>$ $50 \mathrm{~km}$ from Christchurch. A $3 \mathrm{~km} / \mathrm{s}$ surface wave expands to $30.3 \mathrm{~km}$ in $10.1 \mathrm{~s}$ (the mean time to a theoretical alert). If the network had been deployed with more sensors at the perimeter of Christchurch, many of the detection times would have been reduced, and the radius of first alert would have decreased.

A dense QCN network similar to the one deployed in Christchurch, New Zealand is not possible or useful everywhere. QCN requires pre-existing power, Internet, and volunteer computers, so it is limited to populated regions with sufficient resources to afford a base-level cyber-infrastructure. Anecdotally, QCN has found that retaining volunteers may depend on several factors: (1) feedback to the volunteer community to maintain interest, (2) low cost to volunteers of transmitted data per byte, (3) cultural/language differences, or (4) regional groups supporting the effort. For comparison, a year after the $M 8.8$ Maule, Chile, RAMP (Chung et al., 2011) only two participants remained in the region, whereas there were more than 40 RAMP volunteers participating more than $1 \mathrm{yr}$ after the $M 7.2$ Darfield and $M 6.3$ Christchurch, New Zealand, earthquakes.

Perhaps the greatest benefit of a network like QCN is in countries that cannot (or will not) afford a real-time seismic network with traditional research-grade equipment. In such countries, grass roots and academic efforts through cybersocial-seismic networks like QCN may enable real-time earthquake monitoring with $>100$ sensors for less than $\$ 10,000$ in material costs. Because QCN's servers monitor all regions, no regional server, service, or administration is required. In addition, all data collected are available online to access and download. In developing countries like Haiti and Mongolia, Internet usage is as high as $8 \%$ with broadband subscriptions exceeding 1\%, which means $>100,000$ potential cyber-social-seismic network volunteers per country. If only $0.1 \%$ of those potential volunteers are interested in participating, they could host $>100$ sensors in each country.

In the near future, it is likely that cyber-social-seismic networks like QCN will not operate in isolation. By integrating traditional seismic networks with volunteer networks, the combined result will likely exceed both in isolation. Volunteer networks will benefit from the added stability of lownoise traditional sensors in quiet sites, whereas traditional networks will benefit from large quantities of spatially distributed data. Integrating multiple data types such as maximum period of displacement, PGV, and PGA will provide additional constraints.

\section{Conclusion}

The Quake-Catcher Network is designed for lowoverhead installation of dense strong-motion seismic networks capable of providing real-time ground-motion parameters as well as continuous waveform data. The dense RAMP array installed following the 2010 M 7.2 Darfield, New Zealand, earthquake illustrates the potential for low-cost seismic networks to rapidly provide information on earthquake location and magnitude, as well as peak shaking intensities. The expansion of scientific data products resulting from low-cost cybersocial-seismic networks is likely to continue and could provide an important new component of seismic networks to aid in the generation of real-time earthquake products, including ShakeMap (Wald, Quitoriano, Heaton, Kanamori, et al., 1999) and EEW, for moderate to large earthquakes.

\section{Data and Resources}

QCN trigger and waveform data used in this study are available from http://qcn.stanford.edu/sensor/trdl.php (free registration required, last accessed February 2013). Maps of current QCN participants are available from http://qcn .stanford.edu (last accessed February 2013). List and maps of earthquakes detected by QCN are available at http://qcn .stanford.edu/earthquakes (last accessed February 2013). GNS Science earthquake catalog used for comparison to QCN detected events is available from http://magma.geonet .org.nz/quakesearch/ (last accessed December 2013). Some plots were made using Generic Mapping Tools (www.soest .hawaii.edu/gmt, last accessed January 2013; Wessel and Smith, 1991, 1998).

\section{Acknowledgments}

We thank the hundreds of QCN volunteer hosts and the field crew, without whom this study would never have occurred. Many thanks to Dave Anderson whose support and augmentation of BOINC has allowed QCN to grow quickly. We thank Hiroo Kanamori and Dan McNamara for thorough feedback during the revisions of this manuscript. This research was supported in part by NSF EAR 1027802 and the New Zealand Natural Hazards Research Platform.

\section{References}

Allen, R. M. (2006). Probabilistic warning times for earthquake ground shaking in the San Francisco Bay area, Seismol. Res. Lett. 77, 371-376.

Allen, R. M., and H. Kanamori (2003). The potential for earthquake early warning in Southern California, Science 300, 786-789.

Allen, R. M., H. Brown, M. Hellweg, O. Khainovski, P. Lombard, and D. Neuhauser (2009). Real-time earthquake detection and hazard assessment by ElarmS across California, Geophys. Res. Lett. 36, doi: 10.1029/2008GL036766.

Anderson, D. P. (2004). BOINC: A system for public-resource computing and storage, in Proc. of the Fifth IEEE/ACM International Workshop on Grid Computing, Pittsburg, Pennsylvania, 8 November 2004, 4-10.

Anderson, D. P., and J. Kubiatowicz (2002). The world-wide computer, Sci. Am. 286, 40-47.

Campbell, K. W., and Y. Bozorgnia (2008). NGA ground motion model for the geometric mean horizontal component of PGA, PGV, PGD and 5\% damped linear elastic response spectra for periods ranging from 0.01 to 10 s, Earthq. Spectra 24, 139-171.

Christensen, C., T. Aina, and D. Stainforth (2005). The challenge of volunteer computing with lengthy climate model simulations, in First IEEE International Conference on e-Science and Grid Computing, Institute 
of Electrical and Electronics Engineers, Melbourne, Victoria, Australia, 8-5 December 2005, 8-15.

Chung, A. I., C. Neighbors, A. Belmonte, M. Miller, H. H. Sepulveda, C. Christensen, R. S. Jakka, E. S. Cochran, and J. F. Lawrence (2011). The Quake-Catcher Network rapid aftershock mobilization program following the 2010 M 8.8 Maule, Chile earthquake, Seismol. Res. Lett. 82, 526-532.

Clayton, R. W., T. Heaton, M. Chandy, A. K. Krause, M. Kohler, J. Bunn, R. Guy, M. Olson, M. Faulkner, M. Cheng, L. Strand, R. Chandy, D. Obenshain, A. Liu, and M. Aivazis (2011). Community Seismic Network, Ann. Geophys. 54, 738-747.

Cochran, E., J. Lawrence, C. Christensen, and A. Chung (2009). A novel strong-motion seismic network for community participation in earthquake monitoring, IEEE Instrum. Meas. Mag. 12, 8-15.

Cochran, E. S., J. F. Lawrence, C. Christensen, and R. S. Jakka (2009). The Quake-Catcher Network; citizen science expanding seismic horizons, Seismol. Res. Lett. 80, 26-30.

Cochran, E. S., J. F. Lawrence, A. Kaiser, B. Fry, A. I. Chung, and C. Christensen (2011). Comparison between low-cost and traditional MEMS accelerometers: A case study from the $M 7.1$ Darfield, New Zealand, aftershock deployment, Ann. Geophys. 54, 728-737.

Cranswick, E., B. Gardner, R. Banfill, and S. Hammond (1993). Recording ground motions where people live, Eos Trans. AGU 74, 243-244.

Cua, G., and T. H. Heaton (2007). The virtual seismologist (VS) method: A Bayesian approach to earthquake early warning, in Earthquake Early Warning Systems, P. Gasparini, G. Manfredi, and J. Zschau (Editors), Springer, Berlin, Heidelberg.

Donovan, N. C. (1973). Statistical evaluation of strong motion data, including the February 8, 1971, San Fernando earthquake, in Proc. 5th World Conf. Earthquake Engineering, Rome, Italy, 25-29 June 1973, 1252-1261

Earle, P. S., and P. M. Shearer (1994). Characterization of global seismograms using an automatic picking algorithm, Bull. Seismol. Soc. Am. 84, 366-376.

Evans, J. R., R. Allen, A. Chung, E. Cochran, R. Guy, M. Hellweg, and J. Lawrence (2014). Performance of several low-cost accelerometers, Seismol. Res. Lett. 85, doi: 10.1785/0220130091.

Farine, M., N. Thorburn, and D. Mougenot (2004). General application of MEMS sensors for land seismic acquisition-is it time? TLE 23, 246-250.

Gledhill, K., J. Ristau, M. Reyners, B. Fry, C. Holden, and G. Science (2010). The Darfield (Canterbury, New Zealand) $M_{\mathrm{w}} 7.1$ earthquake of September 2010: A preliminary seismological report, Seismol. Res. Lett. 82, 378-386.

Gutenberg, B., and C. F. Richter (1956). Earthquake magnitude, intensity, energy and acceleration, Bull. Seismol. Soc. Am. 46, 105-145.

Heinlein, P., and P. Hartleben (2008). The Book of IMAP: Building a Mail Server with Courier and Cyrus, No Starch Press, San Francisco, California, $352 \mathrm{pp}$.

Holland, J. (2003). Earthquake data recorded by the MEMS accelerometer, Seismol. Res. Lett. 74, 20-26.

Hoshiba, M., O. Kamigaichi, M. Saito, S. Tsukada, and N. Hamada (2008). Earthquake early warning starts nationwide in Japan, Eos Trans. AGU 89, 73-80.

Hughes, L. (1998). Internet E-mail Protocols, Standards and Implementation, Artech House, Boston, Massachusettes, 457 pp.

Kanamori, H. (2005). Real-time seismology and earthquake damage mitigation, Ann. Rev. Earth Planet. Sci. 33, 195-214.

Kilb, D., V. G. Martynov, and F. L. Vernon (2007). Aftershock detection thresholds as a function of time: Results from the ANZA seismic network following the 31 October $2001 M_{1} 5.1$ Anza, California, earthquake, Bull. Seismol. Soc. Am. 97, 280-792.

Luetgert, J. H., J. R. Evans, J. Hamilton, C. R. Hutt, E. G. Jensen, and D. H. Oppenheimer (2009). NetQuakes-A new approach to urban strong-motion seismology (abstract S11B-1707), Am. Geophys. Union Fall Meeting, San Francisco, California, 14-18 December 2009.

McDonough, R. N., and A. D. Whalen (1995). Detection of Signals in Noise, Academic Press, San Diego, California, 495 pp.
McVerry, G. H., J. X. Zhao, N. A. Abrahamson, and P. G. Somerville (2006). New Zealand acceleration response spectrum attenuation relations for crustal and subduction zone earthquakes, Bull. New Zeal. Soc. Earthq. Eng. 39, 1-58.

Wald, D. J., J. V. Quitoriano, T. H. Heaton, and H. Kanamori (1999). Relationship between peak ground acceleration, peak ground velocity, and modified Mercalli intensity for earthquakes in California, Earthq. Spectra 15, 557-564.

Wald, D. J., V. Quitoriano, T. H. Heaton, H. Kanamori, C. W. Scrivner, and C. B. Worden (1999). TriNet "ShakeMaps": Rapid generation of peak ground-motion and intensity maps for earthquakes in Southern California, Earthq. Spectra 15, 537-556.

Wessel, P., and W. H. F. Smith (1991). Free software helps map, and display data, Eos Trans. AGU 72, 441.

Wessel, P., and W. H. F. Smith (1998). New, improved version of generic mapping tools released, Eos Trans. AGU 79, 579.

Zhao, J. X., D. J. Dowrick, and G. H. McVerry (1997). Attenuation of peak ground accelerations in New Zealand earthquakes, Bull. New Zeal. Soc. Earthq. Eng. 3, 133-158.

Stanford University

397 Panama Mall

Mitchell Building 360

Stanford, California 94305

(J.F.L., A.C., C.M.C., J.W.B.)

United States Geological Survey

525 S. Wilson Avenue

Pasadena, California 91106

ecochran@usgs.gov

(E.S.C.)

GNS Science

P.O. Box 30-368

Lower Hutt 5040, New Zealand

(A.K., B.F.)

University of California

Berkeley, 307 McCone Hall

Berkeley, California 94720

(R.A.)

California Institute of Technology

1200 East California Boulevard MC 104-44

Pasadena, California 91125

(T.H., M.D.K.)

Scripps Institution of Oceanography

University of California

San Diego, 9500 Gilman Dr.

La Jolla, California 92093

(D.K.)

University of Delaware

101 Smith Hall

Newark, Delaware 19716

(M.T.)

Manuscript received 8 June 2012;

Published Online 7 January 2014 